\title{
Comparison of EMG power during sleep from the submental and frontalis muscles
}

Daniel J Levendowski'

Erik K St. Louis ${ }^{2}$

Luigi Ferini Strambi ${ }^{3}$

Andrea Galbiati ${ }^{3}$

Philip Westbrook'

Chris Berka'

'Advanced Brain Monitoring, Carlsbad, CA,USA; ${ }^{2}$ Center for Sleep Medicine, Departments of Neurology and Medicine, Mayo Clinic College of Medicine and Science, Rochester, MN, USA; ${ }^{3}$ Department of Clinical Neurosciences, San Raffaele Scientific Institute, Sleep Disorders Center Università Vita-Salute San Raffaele, Milan, Italy
This article was published in the following Dove Press journal:

Nature and Science of Sleep

\begin{abstract}
Objective: Submentalis electromyography ( $s \mathrm{EMG})$ and frontalis electromyography ( $f \mathrm{EMG})$ muscle activities have been used to assist in the staging of sleep and detection of disruptions in sleep. This study was designed to assess the concordance between $s \mathrm{EMG}$ and $f \mathrm{EMG}$ power, by and across sleep stages.
\end{abstract}

Methods: Forty-three records with simultaneous acquisition of differential signals from the submental and frontalis muscles were evaluated. Sleep stages were assigned using the polysomnography signals based on majority agreement of five technicians. The $s$ EMG and $f \mathrm{EMG}$ signals were identically filtered and aligned prior to cross-correlation analysis.

Results: A strong concordance between $s$ EMG and $f$ EMG power was observed, with $95 \%$ of the records exhibiting at least moderate agreement. During rapid eye movement (REM) sleep, $s$ EMG power was significantly less than $f$ EMG power, but exhibited four times greater across-subject variability. $f$ EMG power during wake and non-REM (NREM) sleep was greater than $s$ EMG power, but with 50\% less variability. Differences in wake and N1 mean power and between the other sleep stages were more distinct in the $f$ EMG recordings. Relative changes in $s$ EMG and $f$ EMG power across wake, NREM, and REM stages were essentially identical with median by-subject cross correlations of 0.98 and interquartile ranges of 0.97 and 0.99 , respectively.

Conclusion: The $f \mathrm{EMG}$ and $s$ EMG power values were similar during wakefulness and sleep; however, the frontalis exhibits substantially less between-subject variability. This study established face validity for the use of $f E M G$ in the detection of wake and stages of sleep, and for future applications toward assessment of quantitative REM sleep muscle activity in REM sleep behavior disorder.

Keywords: EMG, frontalis, submentalis, sleep stage, REM sleep without atonia

\section{Introduction}

Acquisition of submental electromyographic activity ( $s \mathrm{EMG}$ ) is recommended in the guidelines of the American Academy of Sleep Medicine for laboratory polysomnography (PSG). ${ }^{1}$ The $s$ EMG signal is used to assist with visual identification of sleep onset, micro-arousals, rapid eye movement (REM) sleep, and REM sleep without atonia (RSWA). ${ }^{2}$ Changes in sleep stage can be recognized by modulations in $s$ EMG amplitude occurring across relatively wide time windows. $s$ EMG has also been shown to be highly sensitive and specific for the recognition and quantification of phasic and tonic muscle activity in REM sleep behavior disorder (RBD). $s$ EMG additionally aids in the distinction between pathologic RSWA diagnostic of RBD and physiologically increased REM sleep muscle activity due to respira-
Correspondence: Daniel J Levendowski Advanced Brain Monitoring, 2237 Faraday Avenue, Suite 100, Carlsbad, CA 92008, USA

Email Dan@b-alert.com 
tory arousals in obstructive sleep apnea (OSA) patients, as well as from normative RSWA levels in patients with OSA without RBD. ${ }^{3-8}$

Frontalis EMG power ( $f$ EMG), derived from frontopolar electroencephalographic (EEG) activity, has been used for the automated staging of sleep and to visually confirm autostaged records. ${ }^{9-12}$ The display of $f$ EMG power relative to the alpha, sigma, and beta power, when used during a focused technical review, contributed to a 5\% improvement in the staging of awake (ie, from $82 \%$ to $87 \%$ ) and a $7 \%$ improvement in the staging of REM sleep (ie, from $73 \%$ to $80 \%$ ). ${ }^{9}$ It has also been demonstrated that EMG activity in the frontalis muscles can identify subclinical abnormalities associated with neurodegenerative disease. ${ }^{13}$

This study was designed to assess the concordance between $s$ EMG and $f$ EMG power with the goal of establishing feasibility and face validity for the use of $f$ EMG power as an alternative to $s$ EMG in a self-applied device that can stage sleep without the chin derivation.

\section{Methods}

\section{Recordings}

Forty-three records with simultaneous acquisition of differential signals from the submental and frontalis muscles with at least 3 hours of PSG recording time were selected for this study. The records were acquired with patient consent under the approval of the Alpha IRB (IRB00006205, protocol 201501) at Complete Sleep Solutions in Murrieta, California, a facility accredited by the American Academy of Sleep Medicine (AASM). Thirty-six records were from subjects referred for a PSG for an assessment of sleep-disordered breathing, and seven were from healthy controls.
The $s$ EMG signals were recorded during laboratory PSG according to AASM criteria using Alice 5 systems (Philips Respironics, Monroeville, PA, USA). The $f$ EMG was extracted from a simultaneously recorded EEG signal acquired with the Sleep Profiler (Advanced Brain Monitoring, Inc., Carlsbad, CA, USA) from sensors located at AF7 and AF8, sampled at $256 \mathrm{~s} / \mathrm{s}$. Figure 1 depicts the location of the Sleep Profiler when worn; written informed consent was obtained from the subject for use of her image. A detailed description of the Sleep Profiler data device and its application during data acquisition was previously provided. ${ }^{9}$

\section{Signal processing}

The $s$ EMG signal was transferred from the PSG record, upsampled using sample repetition to $256 \mathrm{~s} / \mathrm{s}$ and saved into the Sleep Profiler record. The appearance of the primary signals used for this study is presented in Figure 2. The $s$ EMG and

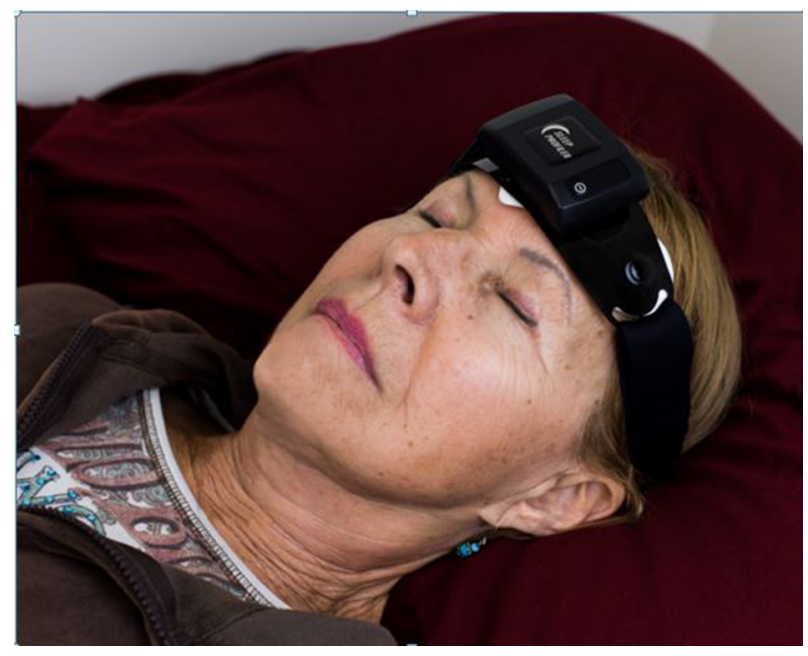

Figure I Subject wearing the sleep profiler.

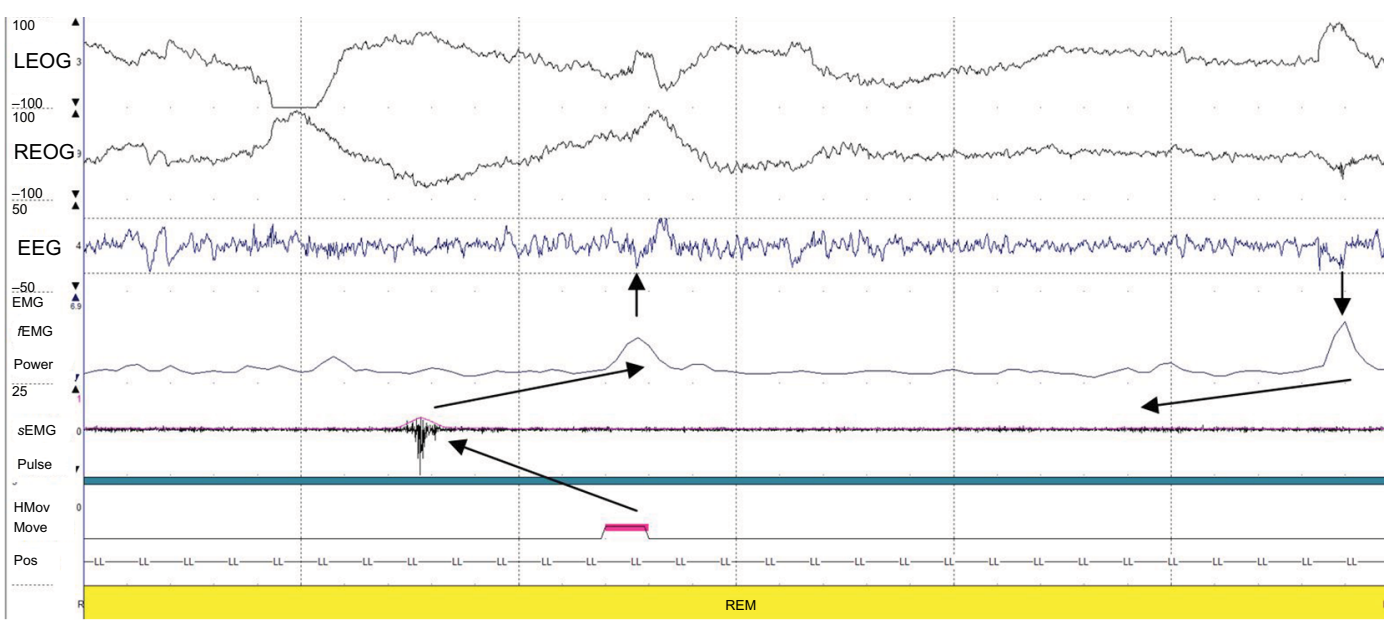

Figure 2 Thirty seconds epoch during REM sleep with differential recordings from AF7-Fpz, AF8-Fpz, and AF7-AF8 (labeled LEOG, REOG, and EEG, respectively), fEMG power, differential EMG, head movement, and head position.

Notes: The first arrow highlights the 4.5 seconds misalignment that coincided with a movement $\sim 2.5$ hour into the study. The second arrow identifies a muscle twitch (eg, facial muscle contraction), based in its appearance in the frontopolar signals but not the submental EMG signals.

Abbreviations: EMG, electromyography; fEMG, frontalis electromyography; REM, rapid eye movement. 
$f$ EMG signals were identically filtered with a $40 \mathrm{~Hz}$ high-pass IIR filter with $61 \mathrm{~Hz},-3 \mathrm{db}$ roll off to derive EMG power $\left(\mu^{2}\right.$ at 1 second epochs). The $s \mathrm{EMG}$ and $f \mathrm{EMG}$ power values were averaged using a 5 second window ( 2 seconds before and 2 seconds after the current sample). Periods were excluded when the PSG power equaled zero (ie, when disconnected during the restroom breaks). One value of $f$ EMG power was then removed every 30 minutes to adjust for misalignment attributed to differences between the hardware clocks. The effectiveness of this transformation was confirmed by comparing values from the first half of each 30 minutes period to the second half of each period.

All studies were visually inspected for artifact that could impact the results; a total of four records were excluded. Two records were excluded due to technically inadequate EEG signal that affected the $f$ EMG power. One record was excluded due to synchronization issues caused by excessive signal loss in the $s$ EMG signal, and another due to uncharacteristic $f$ EMG contamination attributed to breathing artifact (Supplementary material). The descriptive statistics in Table 1, when applicable, were derived from the PSG data.

\section{Data reduction and analyses}

$\mathrm{CC}$ analysis was used to evaluate the temporal relationship between absolute changes in $s$ EMG in comparison with $f$ EMG power. CC across 1 second epochs was used to measure the concordance between $s$ EMG and $f E M G$ power. Next the $s \mathrm{EMG}$ and $f \mathrm{EMG}$ power values were averaged to 15 minute intervals prior to $\mathrm{CC}$ analysis to evaluate individual differences in agreement after smoothing for variability related to sleep stage, microarousals and sleep disordered breathing.

Sleep staging was obtained from the previously reported majority agreement across five raters. ${ }^{9}$ Each record's 1 second $f$ EMG and $s$ EMG power values associated with a consensus sleep stage were averaged. $\mathrm{CC}$ was applied bysubject to the mean power across wake, N1, N2, N3, and REM. In two cases REM sleep was not observed, and in two records N3 was absent. Apnea-hypopnea indexes (AHIs)

Table I Descriptive statistics

\begin{tabular}{|l|l|}
\hline Subjects, $\mathrm{n}$ & 39 \\
Males, $\mathrm{n}(\%)$ & $29(74)$ \\
Age, years, mean (SD) & $44 \pm \mathrm{I} 4$ \\
Recording time, min, mean \pm SD & $37 I \pm 84$ \\
Sleep efficiency, \% & $77 \pm 13.7$ \\
Non-REM time; \% & $83 \pm 7$ \\
REM time, \% & $17 \pm 7$ \\
AHI, events/h & $16 \pm 20$ \\
\hline
\end{tabular}

Abbreviations: $\mathrm{AHI}$, apnea-hypopnea index; REM, rapid eye movement. were scored by one rater, based on AASM 2012 criteria with a $3 \%$ desaturation. ${ }^{1}$

Pearson correlation was used to evaluate the relationship between CC values, sleep efficiency, and AHI. Correlations were stratified by strong $(>0.70)$, moderate $(>0.70$ and $\geq 0.50)$, and weak $(<0.50)$ agreement. $t$-tests were used to identify significant differences in by-stage EMG power.

\section{Results \\ Concordance across I second and I5 minute intervals}

When the 1 second absolute power values were compared, the mean CC across all records was $0.63 ; 36 \%$ of the records exhibiting a strong agreement, $49 \%$ showing moderate agreement, and $15 \%$ displaying weak agreement (Table 2). With the EMG power values averaged into 15 minute increments, the mean CC across all increments in the study was 0.79 . The agreement between $s \mathrm{EMG}$ and $f \mathrm{EMG}$ power was strong in $77 \%$ of the records, moderate in $18 \%$ of the records, and weak in $5 \%$ of the records (Table 2). There was a moderate association between the $\mathrm{AHI}$ and mean 15 minutes $\mathrm{CC}$ values ( $r=0.52, P=0.001$ ), but not with 1 second $\mathrm{CC}$ values.

\section{Concordance by and across sleep stages}

Distributions of the mean across-subject power are presented by stage in Table 3 with the coefficients of variation. Mean $f$ EMG power was greater and with at least $50 \%$ less variability compared to $s$ EMG power during wake and across sleep stages. EMG power progressively decreased for both signals as NREM sleep deepened.

N1 mean power was significantly greater than the REM power for both $s$ EMG and $f$ EMG $(P=0.004$ and 0.0001 , respectively). $s$ EMG and $f$ EMG power during $\mathrm{N} 1$ was at least $30 \%$ greater than that during REM in $87 \%$ of the cases $(32 / 37)$, and both are less in $8 \%$ of the cases $(3 / 37)$. While $s$ EMG power during REM was significantly less than $f$ EMG power, the variability about the mean was four times greater.

Table 2 Distributions of mean CC results stratified by strength of agreement when I second and I5 minutes averaged sEMG and fEMG power was compared

\begin{tabular}{|l|l|l|l|l|l|l|}
\hline \multirow{2}{*}{$\begin{array}{l}\text { EMG power } \\
\text { increment }\end{array}$} & \multicolumn{2}{|l|}{ Strong } & \multicolumn{2}{l|}{ Moderate } & \multicolumn{2}{l|}{ Weak } \\
\cline { 2 - 7 } & $\mathbf{n}$ & $\mathbf{C C}$ & $\mathbf{N}$ & $\mathbf{C C}$ & $\mathbf{n}$ & Mean CC \\
\hline I second & 14 & $77 \pm 4.4$ & 19 & $62 \pm 6.2$ & 6 & $34 \pm 10.7$ \\
\hline 15 minutes & 30 & $87 \pm 7.6$ & 7 & $64 \pm 6.1$ & 2 & $10 \pm 40.3$ \\
\hline
\end{tabular}

Abbreviations: CC, cross-correlation; EMG, electromyography; fEMG, frontalis electromyography; sEMG, submentalis electromyography. 
Table 3 Distributions of power $\left(\mu \mathrm{V}^{2}\right)$ and $\mathrm{CV}$ across all records by stage

\begin{tabular}{|c|c|c|c|c|c|}
\hline \multirow[b]{2}{*}{ Stage } & \multicolumn{2}{|l|}{ sEMG } & \multicolumn{2}{|l|}{ fEMG } & \multirow[b]{2}{*}{$P$-value } \\
\hline & Mean \pm SD & CV (\%) & Mean \pm SD & CV (\%) & \\
\hline Wake & $875 \pm 974$ & 111 & $|50| \pm 794$ & 53 & $<0.01$ \\
\hline $\mathrm{NI}$ & $449 \pm 450$ & 100 & $559 \pm 243$ & 44 & 0.18 \\
\hline N2 & $292 \pm 267$ & 92 & $325 \pm 117$ & 36 & 0.48 \\
\hline N3 & $256 \pm 194$ & 76 & $268 \pm 101$ & 38 & 0.73 \\
\hline REM & $216 \pm 138$ & 64 & $279 \pm 42$ & 15 & 0.01 \\
\hline
\end{tabular}

Abbreviations: CV, coefficient of variation; EMG, electromyography; fEMG, frontalis electromyography; REM, rapid eye movement; sEMG, submentalis electromyography.

As expected, $s E M G$ power was lower during REM sleep; however, the lowest mean $f E M G$ power was observed during N3. There was a moderate concordance between the 37 subjects' $f \mathrm{EMG}$ and $s \mathrm{EMG}$ power values during stage N3 ( $r=0.49, P=0.002)$, likely the result of there being only a $4 \%$ difference in the mean power. The by-stage magnitude of $f$ EMG and $s$ EMG power was unaffected when stratified by age, sex, or OSA (ie, AHI $>10$ ). Both $s$ EMG and $f$ EMG power values when awake were significantly greater than the respective mean power values during $\mathrm{N} 1(P=0.02$ and $<0.0001$, respectively). $s \mathrm{EMG}$ power during N1 was significantly greater than the mean power of $\mathrm{N} 3$ and $\operatorname{REM}(P=0.02$ and $<0.01$, respectively). $f \mathrm{EMG}$ during $\mathrm{N} 1$ was significantly greater than the mean power of N2, N3, and REM (all $P<0.0001$ ), and $f$ EMG power during N2 was significantly greater than REM $(P=0.03)$.

Relative changes in $s E M G$ and $f E M G$ power across wake, NREM, and REM stages for each subject were essentially identical. The median CC was 0.98 with interquartile CC ranges of 0.97 and 0.99 . The gross outlier $(\mathrm{CC}=0.27)$ had an AHI of 109 events/hour and was one of four cases when the $s$ EMG power was greater than $f$ EMG power across all stages of NREM sleep.

\section{Discussion}

We found that temporal changes in EMG measured from the frontalis muscle are highly similar to the submentalis muscle. There was strong concordance between the $s \mathrm{EMG}$ and $f \mathrm{EMG}$ power when smoothed at 15 minute intervals, ie, $95 \%$ of the records exhibited at least moderate agreement, suggesting a trait-like relationship between the two signals during sleep and wake. The 1 second CC confirmed that the signals were measuring similar information but the relationship was influenced by micro-arousals and variability in EMG power during awake periods.

During wake periods, $f E M G$ power exhibited $50 \%$ greater power and $50 \%$ less across-subject variability, suggesting its superiority to $s \mathrm{EMG}$ for purposes of differentiating sleep and wake. This finding likely resulted from differences in interelectrode spacing (ie, $11.3 \mathrm{vs} \sim 4 \mathrm{~cm}$ ). The across-subject variability in $s$ EMG power, being two to four times greater than $f$ EMG power across all stages of NREM and REM, may be attributed to differences in the number of motor units in the frontalis vs submentalis muscles. This explanation cannot be easily confirmed because it is difficult to accurately assess motor unit number in the facial muscles, because the muscles are difficult to activate in isolation. ${ }^{14}$ Alternatively, the divergence in adipocyte distributions over the submentalis muscles, which attenuate the EMG signal, may contribute to the increased across-subject variability. ${ }^{15}$

In clinical practice, $s E M G$ is most often useful in the differentiation of $\mathrm{N} 1$ or drowsy wakefulness from tonic REM and to identify bursts of phasic as well as tonic muscle activity in patients with RBD. ${ }^{16}$ We found that the magnitude of both $s \mathrm{EMG}$ and $f \mathrm{EMG}$ was at least $30 \%$ greater in $\mathrm{N} 1$ as compared to REM in $87 \%$ of the subjects. The influence of sleep stage on EMG power was more apparent in the frontalis recordings. While mean $s$ EMG power during N1 was significantly greater than REM and N3, $f$ EMG power during N1 was significantly greater than REM, N2, and N3, while REM was significantly lower than $\mathrm{N} 2$. The difference in EMG power transitioning from wake to sleep onset was also more clearly defined in the $f \mathrm{EMG}$ signal. These findings suggest that $f \mathrm{EMG}$ is at least non-inferior to $s \mathrm{EMG}$ for use in the staging of sleep in patients without REM-related sleep disorders. ${ }^{9-12}$ Whether an EMG signal with greater stage-related changes in power impacts the detection of RSWA is yet to be demonstrated.

No significant differences in power were observed for either $f \mathrm{EMG}$ or $s \mathrm{EMG}$ when stratified by sleep stage, age, sex, and sleep disordered breathing, unlike what was reported for waking state EEG. ${ }^{17}$ As expected, muscle tone in the submentalis decreased on a continuum and was lowest during REM. The frontalis exhibited a similar decline; however, the cross-subject mean power during REM was slightly greater as compared to N3. One possible explanation for this relative increase in frontalis hyperactivity during REM may be 
a result of facial muscle contractions. Rivera-García et al suggested this increased REM-specific phasic and sustained facial muscle activity was facilitated by limbic activity and may be related to higher emotional activity, a condition that might be exacerbated by RBD. ${ }^{18}$ Despite this potential traitlike influence, it is important to note that the frontalis was less prone to individual differences during REM and exhibited four times less between-subject variability as compared to the submentalis.

Detection of RSWA has been extensively investigated using different muscles and different methods (ie, biceps brachii, extensor digitorum brevis, flexor digitorum superficialis, mentalis, and sternocleidomastoid muscles). ${ }^{2-7,19,20}$ RSWA in the submentalis has been shown to predict the subsequent development of a defined neurodegenerative disease in patients with idiopathic RBD and to correlate with disease severity in Parkinson's disease. ${ }^{21}$ Ferri et al have also reported that the submentalis muscle provides an excellent EMG measure for automated detection of RBD, with subsequent corroboration of automatic and "gold standard" visual RSWA methods., ${ }^{3,4,22}$ It will be interesting to evaluate patients with RBD compared to controls to determine if frontalis muscle activity was overlooked as a potential contributor to future automated methods for the detection of RSWA.

Next to be demonstrated is whether the strong CC between $f \mathrm{EMG}$ and $s \mathrm{EMG}$, presumably driven by muscle activity resulting from spontaneous, movement-related, and/ or respiratory arousal artifact will be apparent in: 1) what is quantified as RSWA for the diagnosis of RBD ie, phasic muscle activity during REM sleep, 2) normal community and sleep apnea controls without dream enactment, or 3) patients receiving antidepressants. ${ }^{2,23-25}$ Studies are currently underway in the USA and Europe to investigate the relationship between $f$ EMG and $s$ EMG, as well as EMG signal recorded from the arms and legs in patients with idiopathic REM behavioral disorder, to determine if $f \mathrm{EMG}$ has sufficient diagnostic accuracy for use in large epidemiological studies for home-based RSWA screening.

\section{Conclusion}

The strong concordance between the $s \mathrm{EMG}$ and $f \mathrm{EMG}$ temporal power confirms feasibility and face validity for the use of $f \mathrm{EMG}$ in the detection of wake and stages of sleep. The transition from wake to sleep as well as sleep stage-related changes in power were more apparent in the frontalis EMG signal. $s$ EMG power was significantly less than $f$ EMG during REM, however, its across-subject variability was four times greater. No significant differences in power were observed for either $f \mathrm{EMG}$ or $s \mathrm{EMG}$ during sleep when stratified by stage and by age, sex, and sleep disordered breathing.

\section{Acknowledgments}

The authors acknowledge Bratislav Velijkovic for his assistance with the signal processing and data extraction, and Dr Delmer Henninger and his staff for their assistance with data acquisition. Levendowski, Berka, and Westbrook were supported by the National Institute of Aging (NIA)-National Institute of Health (NIH) (R44AG050326; R44AG054256). St. Louis received research support from the National Center for Research Resources and the National Center for Advancing Translational Sciences, NIH, through Grant Number 1 UL1 RR024150-01, and the Mayo Clinic Alzheimer's Disease Research Center Grant Award from the NIA (P50 AG016574). The content is solely the responsibility of the authors and does not necessarily represent the official views of the NIH.

\section{Disclosure}

Coauthors Levendowski, Westbrook, and Berka would benefit financially if the Sleep Profiler intellectual property was acquired by a third party. The authors report no other conflicts of interest in this work.

\section{References}

1. Berry RB, Brooks R, Gamaldo CE, et al. For the American Academy of Sleep Medicine. The AASM Manual for Scoring of Sleep and Associated Events: Rules, Terminology and Technical Specification. Version 2.2. Darien, IL: American Academy of Sleep Medicine; 2015.

2. Mccarter SJ, St Louis EK, Duwell EJ, et al. Diagnostic thresholds for quantitative REM sleep phasic burst duration, phasic and tonic muscle activity, and REM atonia index in REM sleep behavior disorder with and without comorbid obstructive sleep apnea. Sleep. 2014;37(10):1649-1662.

3. Ferri R, Manconi M, Plazzi G, et al. A quantitative statistical analysis of the submentalis muscle EMG amplitude during sleep in normal controls and patients with REM sleep behavior disorder. J Sleep Res. 2008;17(1):89-100.

4. Ferri R, Gagnon JF, Postuma RB, Rundo F, Montplaisir JY. Comparison between an automatic and a visual scoring method of the chin muscle tone during rapid eye movement sleep. Sleep Med. 2014;15(6): 661-665.

5. Frauscher B, Iranzo A, Gaig C, et al. Normative EMG values during REM sleep for the diagnosis of REM sleep behavior disorder. Sleep. 2012;35(6):835-847.

6. Lapierre O, Montplaisir J. Polysomnographic features of REM sleep behavior disorder: development of a scoring method. Neurology. 1992;42(7):1371-1374.

7. Montplaisir J, Gagnon JF, Fantini ML, et al. Polysomnographic diagnosis of idiopathic REM sleep behavior disorder. Mov Disord. 2010;25(13):2044-2051.

8. Postuma RB, Gagnon JF, Rompré S, Montplaisir JY. Severity of REM atonia loss in idiopathic REM sleep behavior disorder predicts Parkinson disease. Neurology. 2010;74(3):239-244. 
9. Levendowski DJ, Ferini-Strambi L, Gamaldo C, Cetel M, Rosenberg $\mathrm{R}$, Westbrook PR. The accuracy, night-to-night variability, and stability of frontopolar sleep electroencephalography biomarkers. J Clin Sleep Med. 2017;13(6):791-803.

10. Stepnowsky C, Levendowski D, Popovic D, Ayappa I, Rapoport DM. Scoring accuracy of automated sleep staging from a bipolar electroocular recording compared to manual scoring by multiple raters. Sleep Med. 2013;14(11):1199-1207.

11. Finan PH, Richards JM, Gamaldo CE, et al. Validation of a wireless, self-application, ambulatory electroencephalographic sleep monitoring device in healthy volunteers. J Clin Sleep Med. 2016;12(11):1443-1451.

12. Levendowski DJ, Popovic D, Berka C, Westbrook PR. Retrospective cross-validation of automated sleep staging using electroocular recording in patients with and without sleep disordered breathing. Int Arch Med. 2012;5(1):21.

13. Pan H, Jian F, Lin J, et al. Needle electromyography of the frontalis muscle in patients with amyotrophic lateral sclerosis. Muscle Nerve. 2016;54(6):1093-1096.

14. Patel-Khurana N, Fregosi RF. Motor unit number in a small facial muscle, dilator naris. Exp Brain Res. 2015;233(10):2897-2902.

15. Wan D, Amirlak B, Giessler P, et al. The differing adipocyte morphologies of deep versus superficial midfacial fat compartments: a cadaveric study. Plast Reconstr Surg. 2014;133(5):615e-622.

16. Jeppesen J, Otto M, Frederiksen Y, et al. Observations on muscle activity in REM sleep behavior disorder assessed with a semi-automated scoring algorithm. Clin Neurophysiol. 2018;129(3):541-547.

17. Sella O, Jones RD, Huckabee ML. Age and gender effects on submental motor-evoked potentials. Age. 2014;36(6):9735.
18. Rivera-García AP, Ramírez-Salado I, Corsi-Cabrera M, Calvo JM. Facial muscle activation during sleep and its relation to the rapid eye movements of REM sleep. J Sleep Res. 2011;20(1 Pt 1):82-91.

19. Bliwise DL, Rye DB. Elevated PEM (phasic electromyographic metric) rates identify rapid eye movement behavior disorder patients on nights without behavioral abnormalities. Sleep. 2008;31(6): 853-857.

20. Burns JW, Consens FB, Little RJ, Angell KJ, Gilman S, Chervin RD. EMG variance during polysomnography as an assessment for REM sleep behavior disorder. Sleep. 2007;30(12):1771-1778.

21. Chahine LM, Kauta SR, Daley JT, Cantor CR, Dahodwala N. Surface EMG activity during REM sleep in Parkinson's disease correlates with disease severity. Parkinsonism Relat Disord. 2014;20(7): 766-771.

22. Mccarter SJ, St Louis EK, Sandness DJ, et al. Diagnostic REM sleep muscle activity thresholds in patients with idiopathic REM sleep behavior disorder with and without obstructive sleep apnea. Sleep Med. 2017;33:23-29.

23. Frauscher B, Gabelia D, Mitterling T, et al. Motor events during healthy sleep: a quantitative polysomnographic study. Sleep. 2014;37(4):763-773.

24. Mccarter SJ, St Louis EK, Boeve BF, Sandness DJ, Silber MH. Greatest rapid eye movement sleep atonia loss in men and older age. Ann Clin Transl Neurol. 2014b;1(9):733-738.

25. Ferri R, Bruni O, Fulda S, Zucconi M, Plazzi G. A quantitative analysis of the submentalis muscle electromyographic amplitude during rapid eye movement sleep across the lifespan. J Sleep Res. 2012;21(3):257-263. 


\section{Supplementary material}

The screenshots below present the signal characteristics for a 5 minute epoch and an excerpted 30 second epoch. In both the screenshots, there is a cyclical drift in the LEOG and REOG channels associated with sweat artifact, a pattern that occurs during non-rapid eye movement sleep and when the headband is not properly affixed.

The frontalis electromyography ( $f E M G$ ) power (extracted from the electroencephalographic signal) shows pulsatile changes timed to breathing (likely associated with changes in venous pressure) while the submental EMG signal shows minimal changes in amplitude.
In the lower 30 second screenshot, the changes in $f \mathrm{EMG}$ power are timed to snoring and suggest a breathing rate of $24 \mathrm{bpm}$. There are subtle increases in submentalis electromyography ( $s \mathrm{EMG}$ ) amplitude during some of the periods with loud snoring but the concordance between $f E M G$ and $s$ EMG is compromised.

The automated sleep staging algorithms compensates for large amplitude breathing-related EMG when it occurs in conjunction with loud snoring; however, auto-staging requires a more detailed technical review when the puslating fEMG patterns are apparent.

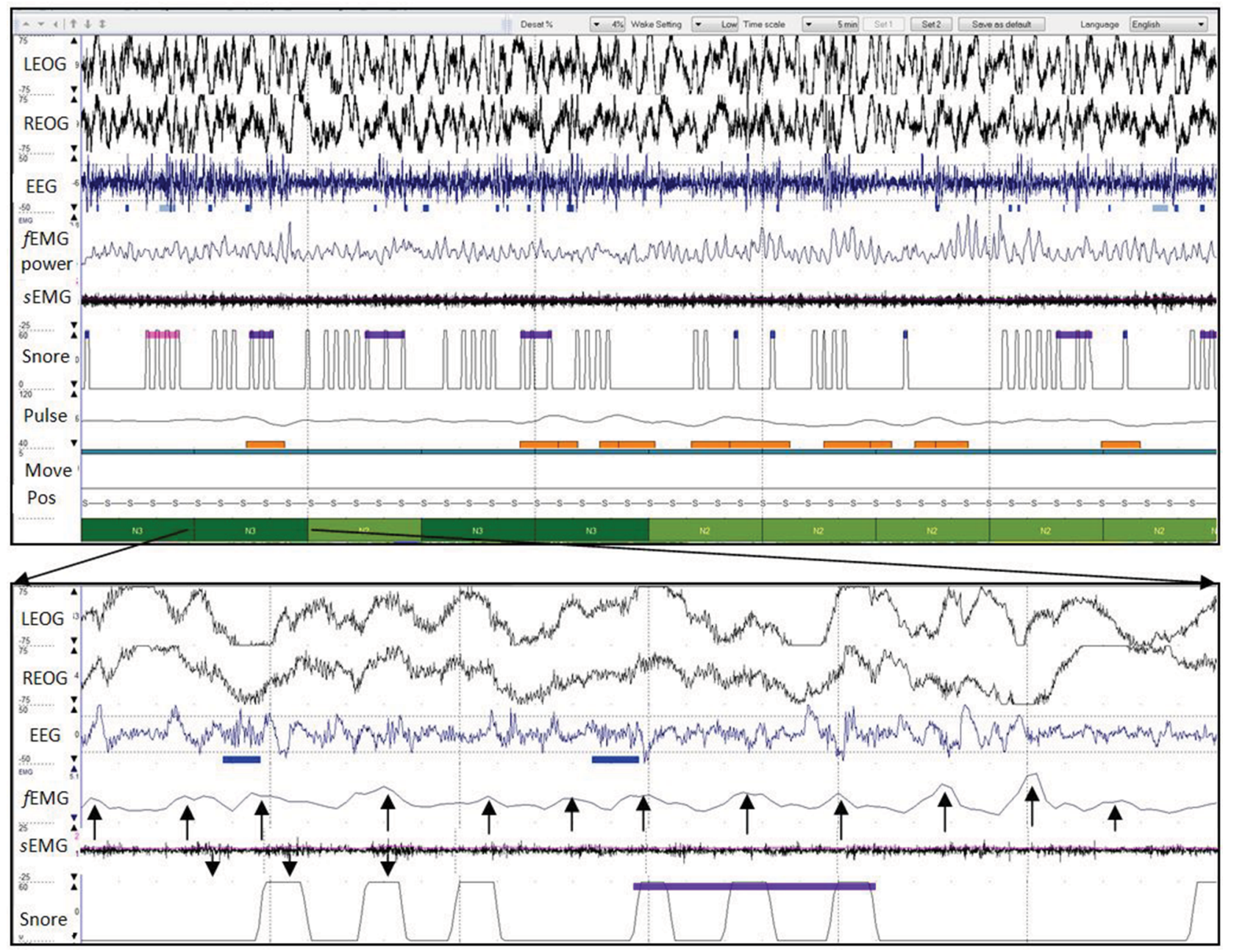

Nature and Science of Sleep

\section{Publish your work in this journal}

Nature and Science of Sleep is an international, peer-reviewed, open access journal covering all aspects of sleep science and sleep medicine, including the neurophysiology and functions of sleep, the genetics of sleep, sleep and society, biological rhythms, dreaming, sleep disorders and therapy, and strategies to optimize healthy sleep. The manuscript

management system is completely online and includes a very quick and fair peer-review system, which is all easy to use. Visit http://www. dovepress.com/testimonials.php to read real quotes from published authors. 